\title{
Johannes Kepler: his place in Astronomy
}

\section{Panagiotis Papaspirou ${ }^{1}$}

University of Athens

Faculty of Physics

Section of Astrophysics, Astronomy

and Mechanics

Panepistimiopolis, Zografos

15784 Athens, Greece

E-mail: p.papaspirou@academy.edu.gr

p.papaspirou973@gmail.com

\begin{abstract}
The place of Johannes Kepler in Astronomy is specified from both directions in historical time. The first refers explicitly to the world - picture of the polymaths and astronomers before his era, and the second to the novel world - picture of the scholars and philosophers that succeeded him. The Hellenic scientific tradition gave birth and shaped explicitly the world - picture of the generations that preceded Kepler's era, and this can be evidently observed in the construction and functions incorporated in the Antikythera Mechanism. Kepler offers a new Paradigm in the science of Astronomy, and contributes greatly to the introduction of a new Paradigm in the Physical Sciences, as well as to the generation of the new philosophical scheme, the one of Mechanistic Philosophy. His great contributions to Astronomy, Mechanics, the Physical Sciences, and Philosophy, which occurs through the introduction of these new Paradigms, rely heavily on the Hellenic, and in particular Alexandrian, tradition of Science and Philosophy. The Antikythera Mechanism can be regarded as the epitome of the Alexandrian tradition in Astronomy, Mathematics and Technology, since it incorporates all these features within a single gear mechanism. The Alexandrian spirit in Philosophy and the Sciences revives in the Age of Renaissance and in the era of Johannes Kepler, and this intellectual fountain serves as a basis for his own great Endeavor in Astronomy and the science of Mechanics, as well as in Optics and Mathematics. Soon after his death the European civilization shall encounter its gigantic transformation through the Age of Enlightenment, the Age of the Industrial Revolution, the Age of Sailing, and its expansion all over the globe. These eras contain as their germ Kepler's contribution to the European civilization.
\end{abstract}

Keywords: History of Astronomy, Scientific Revolution, Paradigm, Renaissance, Newtonian Mechanics, Mechanistic Philosophy, Global Culture.

From Antikythera to the Square Kilometre Array: Lessons from the Ancients, Kerastari, Greece

12-15 June 2012

Speaker 


\section{Introduction.}

The great astronomer Johannes Kepler surely belongs to the Pantheon of the Giants of Science, regarded as one of the prominent thinkers within the framework of the European civilization, a transformer of Astronomy and of the Physical Sciences. As an astronomer, he can be listed among Hipparchus, Ptolemy, Nicolaus Copernicus, Tycho Brahe, Galileo Galilei, and Isaac Newton, as well as Henry Poincare and Albert Einstein [1], [2]. His contributions into the field of Astronomy, and into the field of Cosmology, are related with the structure of Cosmos and the place of Man within it. The scope of his work is not exhausted within the realm of Astronomy, but includes numerous contributions to Mathematics, and Optics as well. Kepler is mostly famous for his three eponymous laws of motion of the celestial bodies, which determine the physical behavior of the planets, as they revolve on their elliptical orbits around the Sun.

Kepler is a strong supporter of the Copernican heliocentric view of the then known Universe, and belongs to the transitory epoch between the naked eye astronomical observations, and the advent of the telescope used for astronomical purposes. The Universe at the times of Kepler included the celestial sphere of the fixed stars, the planets Mercury, Venus, Mars, Jupiter and Saturn, and the Moon. Kepler succeeded to establish the Copernican cosmological doctrine on plausible scientific realistic grounds, but also within the framework of his religious and philosophic thinking. Kepler's creative mind succeeded to formulate explicitly the physical laws named after him, and opened new pathways in all subjects he penetrated [3].

\section{Johannes Kepler and his contribution to the New Paradigms.}

Johannes Kepler introduced a novel Paradigm into the branch of Astronomy, a novelParadigm into the realm of the Physical Sciences [4], and contributed greatly to the generation of the scheme of Mechanistic Philosophy. His work is characterized with the ingenious handling of past scientific concepts, as well as with the introduction of novel scientific concepts. With his attempts he creates a new scientific vocabulary and language. He also introduces a new Methodology, as he struggles to solve novel scientific problems in the field of Astronomy, hence he contributes greatly to the birth of Mechanics. Concrete aspects of his way of thinking serve also as a basis for the Mechanistic philosophy, a novel approach to Nature, Man and God within the European tradition. This tradition influenced greatly the spiritual development of the European civilization [5]. The two Paradigms, the one for the case of Astronomy, and the other, for the case of Physics, are strongly correlated and interrelated with each other, but they might be considered also as distinct. The novel philosophical scheme of Mechanistic Philosophy, which accompanied the development of all Physical Sciences in the Age of Enlightenment and the Age of the Scientific Revolution, incorporates greatly elements of Johannes's Kepler scientific mentality.

The statement of his eponymous three laws of motion for the planets orbiting around the centre of the Universe, the Sun, hence the full establishment of the Copernican heliocentric view of Cosmos, the kinematical and dynamical features and characteristics of the motions of the planets, studied in a detailed extend for the first time, the subsequent abandoning of the Aristotelian Universe, Aristotelian Physics, and of the Ptolemaic Cosmological model, the introduction of new ways for handling with scientific problems, and for posing novel physical questions within the field of Astronomy belong to his greatest achievements. He particularly introduces the concept of "Inertia", related to the mass of the planets, as well as the concept of "Force", which governs the motion on their trajectories around the Sun.

Kepler was both inspired from the spirit of his times, and shared completely its worldview [6], a world - view that incorporates within an organic whole the Christian mystic tradition and the Neoplatonic and Neopythagorean elements in Philosophy and Science. 


\section{The scientific work of Johannes Kepler}

Johannes Kepler formulated his eponymous laws which govern the motion of the planets around the Sun. According to the first law, the trajectories of the planets have the form of ellipses, with the Sun centered at the one focus of the ellipse. According to the second law, the planets move in such a manner, so that the line joining them with the Sun sweeps equal areas in equal time intervals. The third law states the exact relationship between the square of the orbital period of the planets and the cube of the semimajor axis of their ellipse, which is the same for all the planets. We can derive easily these three laws, starting from the three Newtonian Axioms of Motion and the Newtonian law of Gravitational interaction.

These laws permitted further the justification of Newton's Paradigm in Physics and the arising of the New Physics. The stream of the "Newtonian consciousness", carried by the so called "Newtonians", departs entirely from the Aristotelian tradition in a dramatic way, and destroys for ever the foundations of the Medieval and Renaissance Mentality, thus setting the "noospheric" basis of our Modern World [7].

Kepler's work exceeds the level of a just very clever fitting of the astronomical observational data he had at hand to some known curve. He introduces for the first time, and on empirical grounds, the novel geometric shape of the planetary orbits, the ellipses, and reveals the underlying Physics of the celestial motions.

The data he possessed were the fruits of the lifelong work of another giant of Astronomy, Tycho Brahe the most accurate ephemerides and astronomical tables ever composed in the history of European Astronomy up to the age of Kepler. The history of this astronomical observational tradition starts already within the realm of the Hellenic tradition, passes on to the Hellenistic Age of Claudius Ptolemy, continues through the Byzantine civilization, and the Islamic Golden Age, reaching up to the age of Copernicus and of Kepler. Tycho Brahe immediately acknowledged the astronomical, mathematical and scientific genius of Johannes Kepler, and particularly insisted of having Kepler as his collaborator in Tycho's new observatory, in Prague [8].

Other contributions of Johannes Kepler include the invention of a primitive form of differential and integral calculus, based on the work of Archimedes [9], in order to compute areas and volumes of solids of revolution, the extensive use of logarithms, the invention of a new type of refractive telescope, the "Keplerian telescope", the first modern physical foundation of the science of Optics, the study of the properties of some of the so - called "Kepler - Poinsot polyhedra", a novel type of a plane - filling arrangement, and the statement of his famous eponymous Conjecture about the densest packing of spheres in space [10].

\section{Defining Johannes's Kepler place in Astronomy}

Johannes Kepler lives within the epoch of an "Interregnum", a transient phase of the European civilization between the old religious, philosophical, technological and scientific world of Medieval Europe and Renaissance, and the novel forthcoming world, the "Mechanized" one. The old Zeitgeist is being replaced by new concepts, by a novel corpus of scientific languages, theories, methodologies, and by novel technologies. At the same time, new branches of philosophical systems, novel forms of the Fine Arts, of religious thought, and in general, novel communicative fields of social structures, appear, while Science and Technology blossoms.

We can trace the pathways which sustained Kepler's "Weltanschauung" in two directions in historical time, with point of reference his era. The one strives towards the past, and the other towards the future. The first pathway of ideas, concepts and mental worlds starts already in the Hellenic culture, and its tradition in Philosophy, the Physical Sciences, Astronomy and Mathematics. In its acme the Hellenic culture produced wonderful scientific 
instruments, such as the Antikythera Mechanism. The Antikythera Mechanism, as a scientific artifact, was unsurpassed for centuries. This wide river of philosophical perceptions of Reality and Science, starts from the Hellenic civilization, and the Hellenistic era in particular, and continues its flow to the Byzantine civilization. Then, it spreads and blossoms during the Golden Age of the Islamic civilization, and finally returns to Europe through the massive translations of texts from the Arabic and Greek into Latin, or by the current of Byzantine exiles finding refugee in the European ground. This energetic group of scholars and polymaths, on their own Exodus, carry the scientific and philosophical heritage we mention. Their counterpart polymaths of the Islamic scientific heritage played also such a most significant historical role for the transmission and development of knowledge. The "Little Renaissance" of the 12th century, and the Age of Renaissance in the 14th century in Europe, both seem to originate from this fountain of Knowledge and great Personalities [11], [12].

The Greek civilization and culture, already from the epoch of flourishing and blossoming of Astronomy, that is at least from the epoch of Archimedes, which can also be regarded as the founder of an active astronomical school in Syracuse, contributes greatly to the scientific understanding of the celestial phenomena. These remarkable theoretical and technological achievements were crowned with astronomical and cosmological theories, as well as detailed astronomical observations. Tangible and intangible astronomical instruments artifacts are constructed, such as mural instruments, types of dioptras, of armillary spheres, of parallactic instruments, of astrolabes, and astronomical mechanisms akin to the Antikythera Mechanism. These reproduced the motions of the heavenly bodies within the standards of great accuracy, and based on specific physical and mathematical laws. The Antikythera Mechanism, this most famous example of Greek innovation in Science and Technology, stands as one of the most ingeniously constructed astronomical instruments ever built [13], [14], [15].

In the forthcoming ages of the European thought the dogmas of the Christian religion are fused with the doctrines of the Greek philosophical view of cosmos. An exceptional landmark in this European tradition is Nicolaus Cusanus, a follower and prolific writer belonging to the Christian mystic Neoplatonic tradition. Cusanus is an ecclesiastical reformer and cardinal, a kind of personality which influenced greatly Johannes Kepler by his philosophical and cosmological views [16]. We mention that the Neoplatonic school of thought comprises a long - lived tradition in the history of Philosophy and serves as a profound basis for understanding the Universe, its creation and origin, and the laws that govern this unified, organic whole. Cosmos is stratified into a strict hierarchical multi- level structure of varying degrees of complexity, and obeys mathematical harmonies and symmetries, expressed within the realm of teleological arguments and the qualitative and quantitative investigation of these symmetries and harmonies. These qualitative and quantitative characteristics of Cosmos are inherited as Platonic Forms, stemming from the spirit of the craftsman of Cosmos. This "craftsman" in the Platonic and Neoplatonic tradition is replaced by the Christian God, who interpenetrates all forms and aspects of natural existence, thus completing the divine order in the Universe [17].

According to the idiosyncratic doctrines of Nicolaus Cusanus, and his exploitation regarding the most important categorical realities endowed within the structure of the Universe, that is God, Nature, Christ and Humanity, the Earth does not stay fixed at some point, nor can it be regarded as the physical center of the Universe. In addition, the celestial spheres emerging naturally within the Aristotelian - Ptolemaic cosmological model do not attain an exactly spherical form. According to Cusanus, God is everywhere and nowhere, He is the center and the circumference of the Universe. Jesus Christ, the God - man, is the mediator between the God and his Creation, the connection with the Divine Order. We remark that the religious stream of thoughts within the Christian tradition offers for example a way for the Salvation of the Souls to become possible, whereas the scientific elements of Kepler's thought have as their origin the 
long and complex thread, which starts from the begin of the Hellenic civilization, and the birth of the Science of Astronomy.

The Ratio and the Intellectus of the human mind, that is his Dianoia and Noesis, is capable of knowing by measuring the objects of our knowledge, while being assimilated in some way to them. This insistence for measuring may be regarded as one of the leading leitmotivs in Johannes's Kepler thought. Also, the abandoning of the exactness of the Aristotelian - Ptolemaic cosmological model becomes more than evident, and encourages the deeply religious astronomer on his own personal path.

The other direction starts from the age of Kepler and the Zeitgeist of his era and his cotemporaries, and strives towards the future, to the upcoming "Mechanization of the world picture". This is followed also with the advent of the "mechanistic mentality" into Science and Philosophy, mainly through the works of Newton, Leibniz, Descartes, and Spinoza. It gives birth to the Age of Enlightenment, the Age of Sailing, and the Industrial Revolution [18], [19]. New philosophical schemata and Paradigms in science are created within this new mental atmosphere of bold revolutionary thinkers. The perceptions of God, Man, and Cosmos, as well as of Society, and their mutual interrelations and interactions are redefined, and shaped within the novel social and industrial structures [20]. Thus, we have to consider the birth of the New Astronomy, of Modern Physics, and of Mechanistic Philosophy, which shape the technological, methodological, epistemological, and ontological frameworks of reference. Such a detailed analysis has to be furthermore investigated by taking into account the most general sociological relations between these social structures and levels of discourse [21], [22], [23], [24].

\section{Johannes Kepler and his own path towards creating Science}

Kepler proposes successfully a new kind of theoretical model in Astronomy, struggling at first to define the exact shape of the orbit of Mars, the outer planet that exhibits the most irregular behavior on his route on the celestial sphere [7]. He introduces a completely new astronomical model, which abandons forever the necessity of the uniform cyclic motion. The old way for performing Astronomy regards as the only permissible motions of the celestial bodies strict combinations of eternal and perfect circular motions. Kepler replaces them with the elliptic motion. He establishes the Copernican heliocentric cosmological model on completely new epistemological grounds, and extends its features, the global and the local ones. He attacks reality from all sides, the theological, the ontological, the epistemological, the methodological, and strongly believes that he reveals the secrets of Creation in his monumental work.

The Copernican system, within the work of Johannes Kepler, is not merely a convenient mathematical device for "saving the phenomena", just a set of convenient mathematical rules and recipes for describing the features of the heavenly bodies, thus lacking completely a truly realistic scientific basis. The tradition of "saving the phenomena" starts already from the era of Ptolemy, and continues on to all the subsequent generations of Hellenistic, Byzantine, Muslim, and European astronomers. It is remarkable that many of these polymaths tried to abandon the philosophically unjustified concept of the equant within the Ptolemaic model, since the notion of the equant destroys the full validity of the principle of the uniform cyclical motion. [25].

The consequences of the acceptance of the heliocentric model of Copernicus are not trivial. The novel model is heliocentric and not geocentric. Thus, Earth becomes just a planet and not the center of the Universe, Cosmos itself becomes a vast space, perhaps of infinite dimensions, Astronomy is characterized by a novel conceptual structure, the astronomical terms defined acquire a new meaning and a new function within this new theory. Here, we always have to acknowledge that Kepler bases his work on Neoplatonic and Neopythagorean philosophical beliefs and concepts, and on the evidence of apex theoretical ideas and technology [7]. 
Johannes Kepler defends the Copernican system, and places it to the status of the unique physical model. Thus, he introduces a novel mentality of how to handle with physical problems, and in particular with astronomical problems, and a novel way of asking scientific questions, as well as new ways of answering these questions and solving the generated scientific riddles. He combines his effort with the detailed investigation of the empirically obtained astronomical observational data, and with the help of the already existing or newly devised mathematical machinery. Kepler's insistence about unraveling the secrets of Creation is based on a strictly quantitatively and abstract mathematical manner. Among others, he introduces into the field of Astronomy novel mechanical concepts, such as the inertia of the masses of the planets, a kind of gravitational force stemming from the Sun and affecting the celestial objects, influencing continuously their movement on their predefined elliptical orbits [16].Thus, he starts asking new types of questions, and poses new problems to be solved [25].

He wonders why the Sun must be placed in the center of the Universe, and answers this question based on philosophical and epistemological grounds. He wonders why the planets move around the Sun, and tries to define the shape of their trajectories. He wonders, perhaps for the first time, what kind of physical force is responsible for keeping them on their orbits. Then, he tries to determine their further kinematical and dynamical characteristics. This new kind of force is similar to the magnetic force, as described in the most influential work of Gilbert, the "De Magnete" [26]. This dynamical physical explanation stays as the Prototype for the notion of a physical Force, and is incorporated into the Newtonian Picture of Cosmos [27], [28]. The physical Reality in his work is conceivable within the limits of human understanding, and its properties are measurable, obeying various kinds of symmetries and harmonies. This strong tendency to the exact mathematical and empirical description of the astronomical phenomena is one major feature of the new Paradigm in Astronomy and in the Newtonian Mechanical Paradigm, and also serves as a solid foundation for the Mechanistic philosophy, as introduced by Johannes Kepler by Galileo Galilei [29]. In his struggle, Johannes Kepler uses the mathematical method of exhaustion of Archimedes in order to measure areas and volumes, hence he expands the mathematical universe of the Physical Sciences [9]. The nature of all these scientific questions is groundbreaking and surpasses by far the geometrical, static Scholastic way of thinking. It is remarkable that Johannes's Kepler first monumental work in Astronomy, the "Mysterium Cosmographicum", which can be considered both as one of the most beautiful physical models ever conceived, and as one of the most unsuccessful attempts of describing Creation, contains the thread of questions he tries to solve within the realm of his following works. In this first rudimentary work the great astronomer is puzzled over why there are only six planets. He also attempts to justify why their orbits and their velocities obtain the specifically concrete values proposed in Nicolaus's Copernicus work "De Revolutionibus", as well as why the Sun must be regarded as the center of the Universe [1].

Another important component of Kepler's Zeitgeist is the relation among the scientific community, composed by scholars and polymaths, and educated in monastic schools or concrete institutions, such as universities, and the policies of the Church. The reaction against the work of Copernicus had an immense character, as well as against the work of Galileo. The Old world - view acquired the status of a dogma, and was based solely on the Aristotelian Paradigm and the authority of the Holy Scripts [29]. This transformation of the world - view led to a most significant, transformation within the realm of the European societies, and marked the expanding freedom from the constraints of the Old World.

Johannes Kepler chooses a particular physical and mathematical model, not only by mere rational inspection of all models or theories beforehand, but also driven by theological, philosophical and aesthetic beliefs. This way of thinking corresponds entirely to the spirit of his era, especially the Neoplatonic one, as expressed by Plato's "Timaeus" [30].He is a devoted seeker of truth, and reaches the borders of the already known, his mental reflexes become even more refined and sensitive. He learns to observe reality and experience and evaluates the 
empirical as well as theoretical aspects of the world in their most fine detail. The echo of his achievements thunders throughout the generations up to our age of Post-modernity and the Global Heritage of Astronomy.

\section{Conclusion}

Johannes Kepler shares a longstanding tradition in Astronomy, which roots can be traced to the Hellenic Alexandrian Philosophy and Science, and to giants of Science, such as Archimedes, Hipparchus, and Ptolemy. The Hellenistic era and tradition of the Physical Sciences, and Astronomy in particular, formed the framework and the scientific mentality of Johannes Kepler. We can immediately recognize, after examining the structure or the functions of the Antikythera Mechanism, the great advances in Astronomy and Science within this culture, that form a thread uniting Kepler's thought and the Antikythera Mechanism. Kepler is the offspring of the Hellenic tradition in Science, and he is the driving force for the generation of several novel Paradigms. His work finally places Astronomy, and the Physical Sciences, within the realm of the Global Cultural Heritage, linking the Hellenic tradition with today's astronomical satellite and spacecraft missions dedicated for the exploration of the Universe, as well as with all the fields of Observational Astronomy.

\section{References}

[1] M. Caspar, Kepler, New York: Courier Dover Publications, 1993.

[2] H. Goldstein, C. P. Poole, J. L. Safko, Classical Mechanics, $3^{\text {rd }}$ ed. , Addison Wesley, 2002.

[3] P. Casini, (1988) "Newton's Principia and the Philosophers of the Enlightenment", Notes and Records of the Royal Society of London 42 (1): 35-52.

[4] T. S. Kuhn, The Structure of Scientific Revolutions, 3rd ed. Chicago, IL: University of Chicago Press, 1996.

[5] M. M. Munnynck, (1911), Mechanism., In The Catholic Encyclopedia. New York: Robert Appleton Company. Retrieved August 27, 2012 from New Advent: http://www.newadvent.org/cathen/10100a.htm

[6] A. Koestler, The Watershed: a Biography of Johannes Kepler, Garden City: Doubleday, 1960.

[7] J. L. E. Dreyer, A History of Astronomy from Thales to Kepler, Dover Publications Inc, 1967.

[8] K. Ferguson, The nobleman and his housedog: Tycho Brahe and Johannes Kepler: the strange partnership that revolutionized science. London: Review, 2002.

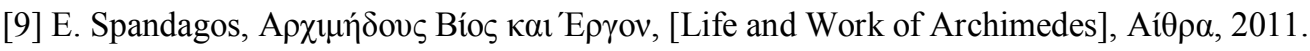

[10] J. Kepler and C. Frisch, Joannis Kepleri Astronomi Opera Omnia (John Kepler, Astronomer; Complete Works), 8 vols.(1858-1871), Francofurti a.M.et Erlangae, Heyder \& Zimmer.

[11] E. Theodossiou, E. Danezis, V.N. Manimanis and E.-M. Kalyva, From Pythagoreans to Kepler: The dispute between the geocentric and the heliocentric system, Journal of Astronomical History and Heritage JAH2 Vol. 5, No. 1, June 2002, pp. 89-98.

[12] E. Theodossiou, V.N. Manimanis and M. Dimitrijević, The contribution of Byzantine priests in astronomy and cosmology. I. The Church Fathers: The Three Bishops St. Basil the Great, St. Gregory of Nazianzus and St. John Chrysostom, European Journal of Science and Theology, EJST, vol. 7, No. 2, 
June 2011, pp. 57-69.

[13] T. Freeth, Y. Bitsakis, X. Moussas, J. Seiradakis, A. Tselikas, H. Mangou, M. Zafeiropoulou, R. Hadland, D. Bate, A. Ramsey, et al., Decoding the ancient Greek astronomical calculator known as the Antikythera Mechanism, Nature, 11/2006, V. 444, p.587-591, (2006).

[14] X. Moussas, The Antikythera Mechanism: A Mechanical Cosmos and an Eternal Prototype for Modelling and Paradigm Study, Adapting Historical Knowledge Production to the Classroom, Section A, 113-128, 2010.

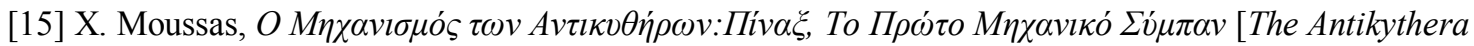

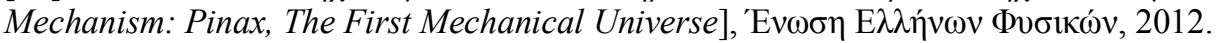

[16] C. L. Miller, "Cusanus, Nicolaus [Nicolas of Cusa]", The Stanford Encyclopedia of Philosophy (Fall 2009 Edition), Edward N. Zalta (ed.),URL=<http://plato.stanford.edu/archives/fall2009/entries/cusanus/>.

[17] Siorvanes, L., 1996, Proclus. Neo-Platonic Philosophy and Science, New Haven: Yale University Press.

[18] E. Cassirer, The Philosophy of the Enlightenment, tr. Fritz C.A. Koelln and James P. Pettegrove, Boston: Beacon, 1955.

[19] T. S. Ashton, The Industrial Revolution (1760-183), Oxford University Press, 1948.

[20] F. Novotny, Painting and Sculpture in Europe, 1780-1880 (Pelican History of Art), Yale University Press, 2nd edn. 1971

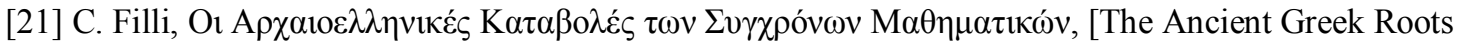

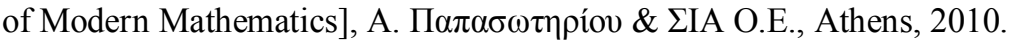

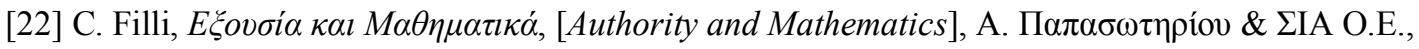
Athens, 2009.

[23] E. Bitsakis, Physique contemporaine et matérialisme dialectique, Ediotions Sociales, Paris 1973 -

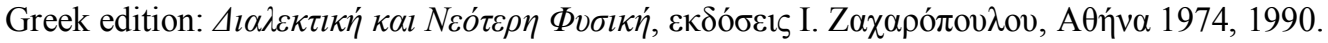

[24] E. Bitsakis, La nature dans la pensée dialectique, Editions Sociales, Paris 1974 - Greek edition: $H$

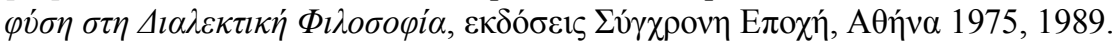

[25] T. Kuhn, The Copernican Revolution. Harvard University Press, 1957, (copyright renewed 1985)

[26] J. P. Derek, ed. (Facsimile of 1900 Thompson translation). William Gilbert: On the Magnet. The Collector's Series in Science. New York: Basic Books, 1958

[27] D. Gentner, S. Brem, R. Ferguson, P. Wolff, T. B. Ward (Ed), S. M. Smith (Ed), J. Vaid (Ed), Analogy and creativity in the works of Johannes Kepler, Gentner, Creative thought: An investigation of conceptual structures and processes, Washington, DC, US: American Psychological Association, 1997.

[28] Max Jammer, Concepts of Force: A Study in the Foundations of Dynamics, Cambridge (Mass): Harvard U.P., 1957 New York: Harper, 1962 New York: Dover, 1999.

[29] Taton, René; Wilson, Curtis, eds. , Planetary astronomy from the Renaissance to the rise of astrophysics Part A: Tycho Brahe to Newton. Cambridge: Cambridge University Press, 1989.

[30] G. Vlastos, Plato's Universe, Seattle: University of Washington Press, 1975. 